\title{
Hydrogen desorption kinetics from H-Si (111) surfaces studied by optical sum frequency generation and second harmonic generation
}

\author{
Q2 Q8 Md. Abdus Sattar, ${ }^{a}$ Khuat Thi Thu Hien, ${ }^{a}$ Yoshihiro Miyauchi, ${ }^{b}$ \\ Goro Mizutani $^{\mathrm{a} *}$ and Harvey N. Rutt ${ }^{\mathrm{c}}$
}

We have studied hydrogen desorption from a flat H-Si (111) $1 \times 1$ surface at $711 \mathrm{~K}$ by observing sum frequency generation (SFG) and second harmonic generation (SHG) spectra. Flat H-Si (111) surfaces were prepared by dosing hydrogen molecules in a Q3 ultra-high vacuum chamber with a base pressure of $10^{-8} \mathrm{~Pa}$. Combining the SFG and SHG methods, the desorption order has been clarified over the whole hydrogen coverage range from 1 monolayer (ML) to $0 \mathrm{ML}$. The hydrogen desorption was assigned as second order in the high coverage range of $1 \mathrm{ML}-0.18 \mathrm{ML}$ by using SFG spectroscopy and as first order in the coverage range of $0.18 \mathrm{ML}-0.0 \mathrm{ML}$ by using SHG spectroscopy. Copyright $\odot 2016$ John Wiley \& Sons, Ltd.

Keywords: sum frequency generation (SFG); second harmonic generation (SHG); Si (111)1×1 surface; desorption order; hydrogen coverage

\section{Introduction}

Hydrogenated flat Si (111) surfaces are attractive substrates for silicon-based devices. The hydrogen desorption process from the Si surface plays an important role in the epitaxial growth of $\mathrm{Si}$ by chemical vapor deposition. ${ }^{[1]}$ For this reason, the hydrogen desorption process has been investigated by various methods such as laser-induced thermal desorption (LITD), ${ }^{[1,2]}$ second harmonic generation (SHG) spectroscopy, ${ }_{1}^{[3]}$ in situ surface infrared (IR) spectroscopy, ${ }_{1}^{[4]}$ scanning tunneling microscopy, ${ }_{1}^{[5]}$ and temperature-programmed desorption, ${ }^{[6]}$ and so on. The hydrogen desorption from a Si (111)7 $\times 7$ surface was studied by using LITD by B.G. Koehler and his coworkers. They suggested that the desorption order of hydrogen molecules above 0.2 monolayer (ML) displayed second-order kinetics. ${ }^{[1]}$ Gupta et al. studied hydrogen desorption by temperature-programmed desorption, and they revealed that on the $\mathrm{H}-\mathrm{Si}(111) 1 \times 1$ surface hydrogen atoms started to desorb at $720 \mathrm{~K}$ and completely desorbed at $800 \mathrm{~K}^{[7]}$ They also suggested that the desorption of hydrogen molecules for monohydride species follows second-order kinetics. These suggestions are consistent with other groups such as B.G. Koehler et al. ${ }^{[1]}$ Hien et al. studied isothermal hydrogen desorption from a H-Si (111) $1 \times 1$ surface prepared by chemical etching by using sum frequency generation (SFG) spectroscopy at different temperatures. They suggested that the hydrogen desorbed homogeneously, but they could not decide the desorption order at hydrogen coverage below $0.2 \mathrm{ML}^{[8]}$ because at the coverage lower than $0.2 \mathrm{ML}$, the resonant SFG signal was indistinguishable from the non-resonance background. The surface coverage corresponding to $\sim 1 \mathrm{ML}$ is the maximum surface density of the hydrogen atoms on the Si (111) surface, namely, $1 \mathrm{ML}=7.8 \times 10^{14}$ (atoms $\left./ \mathrm{cm}^{2}\right)^{[9,10]}$

The aforementioned works show that hydrogen desorption from the single crystal Si (111) surface has been studied with interest for a long time. However, some unanswered problems about the desorption order still remain, especially at low hydrogen coverage. The desorption order for coverage below $0.2 \mathrm{ML}$ over a range of temperature of $680 \mathrm{~K}<\mathrm{T}<800 \mathrm{~K}$ was investigated using SHG by Reiders et al. only. ${ }^{[3]}$ They found that the result can be adequately characterized by an intermediate reaction order of $1.5 \pm 0.2$.

In our study, we investigated the hydrogen desorption mechanism from a flat $\mathrm{H}-\mathrm{Si}(111) 1 \times 1$ surface at $711 \mathrm{~K}$ by using both SFG and SHG spectroscopy. We have used SFG spectroscopy for investigating the hydrogen desorption at high hydrogen coverage, and SHG for low hydrogen coverage on the hydrogenated Si (111) surfaces. In our SHG experiment, the sample was heated for a much longer time than Reiders et al. in order that the fitting of the desorption curve should be much more exact. ${ }^{[3]}$

Nonlinear optical spectroscopies such as SFG and SHG techniques are used as useful nondestructive and sensitive tools to study the properties of surfaces and interfaces. SFG is a secondorder nonlinear optical process. In SFG, two photons with different frequencies of visible $\left(\omega_{1}\right)$ and IR $\left(\omega_{2}\right)$ interact with a nonlinear medium simultaneously to generate a photon with the sum of

* Correspondence to: Goro Mizutani, School of Materials Science, Japan Advanced Institute of Science and Technology, 1-1 Asahidai, Nomi, Ishikawa 923-1292, Japan.

E-mail: mizutani@jaist.ac.jp

a School of Materials Science, Japan Advanced Institute of Science and Technology, 1-1 Asahidai, Nomi, Ishikawa, 923-1292, Japan

b Department of Applied physics, National Defense Academy of Japan, Hashirimizu 1-10-20, Yokosuka, Kanagawa, 239-8686, Japan

c School of Electronics and Computer Science, University of Southampton, Southampton SO17 1BJ, UK 
the two frequencies $\omega_{1}+\omega_{2}$. SHG is a special case of SFG with $\omega_{1}=\omega_{2}=\omega$ and a photon with the frequency of $2 \omega$ is generated. In this study, SFG spectroscopy was used to observe the vibrational spectrum of an $\mathrm{H}$-Si surface before and after heating at $711 \mathrm{~K}$. A SFG signal could be obtained with hydrogen coverage from $1 \mathrm{ML}$ to $0.2 \mathrm{ML}$. When hydrogen coverage became low $(\sim 0.2 \mathrm{ML})$, the SFG signal was close to the background, and the vibrational mode could not be seen. On the other hand, SHG was very sensitive to dangling bonds on the surface. ${ }^{[3]}$ Therefore, we applied SHG spectroscopy to measure the remaining hydrogen coverage when the coverage was low. Then, the desorption order could be clarified in the whole coverage range from $0 \mathrm{ML}$ to $1 \mathrm{ML}$.

\section{Experimental setup}

Si (111) substrates $\left(25 \times 5 \times 0.1 \mathrm{~mm}^{3}\right.$ in dimension) were cut from $\mathrm{N}$ type flat Si wafers. The resistivity of the samples was $1 \sim 5 \Omega \mathrm{cm}$. Sample preparation and hydrogen termination were reported in detail by Hien et al. ${ }^{[8]}$ Here, we will describe in detail the SFG and

F1 SHG measurements. The optical system is shown in Fig. 1. For SFG measurement, we used doubled-frequency pulses at wavelength $532 \mathrm{~nm}$ with photon energy of $\sim 2.33 \mathrm{eV}$ generated by a modelocked $\mathrm{Nd}^{3+}$ : YAG laser operating at a repetition rate of $10 \mathrm{~Hz}$ and a pulse width of $30 \mathrm{ps}$ as the incident visible light. Tunable infrared light pulses output from an optical parametric generator

Q4 with an amplifier (optical parametric generator/optical parametric

Q5 amplifier) system were used as the incident IR light. The incident visible light was passed through a Glan polarizer, a band pass filter, a lens with focal length $f=300 \mathrm{~mm}$, and a $\mathrm{CaF}_{2}$ window of the UHV chamber with a pulse energy of $\sim 15 \mu \mathrm{J} /$ pulse. The IR light was focused by a $\mathrm{CaF}_{2}$ lens with a focal length of $f=250 \mathrm{~mm}$ with the pulse energy of $\sim 70 \mu \mathrm{J} /$ pulse at the sample. The angles of incidence of the visible and IR light beams were $\sim 45^{\circ}$ and $60^{\circ}$, respectively. A delay line was used to adjust the temporal overlap of the IR and the visible pulses at the sample. The SFG light generated from the sample in the reflective direction was passed through a glass window of the chamber, band pass filters (Asahi SV0490), a polarizer plate (Sigma Koki, SPF-30C-32), and finally the SFG signal was focused onto the monochromator entrance by a lens and was detected by a photomultiplier. The SFG signals were obtained

\section{Optical setup for SFG \& SHG}

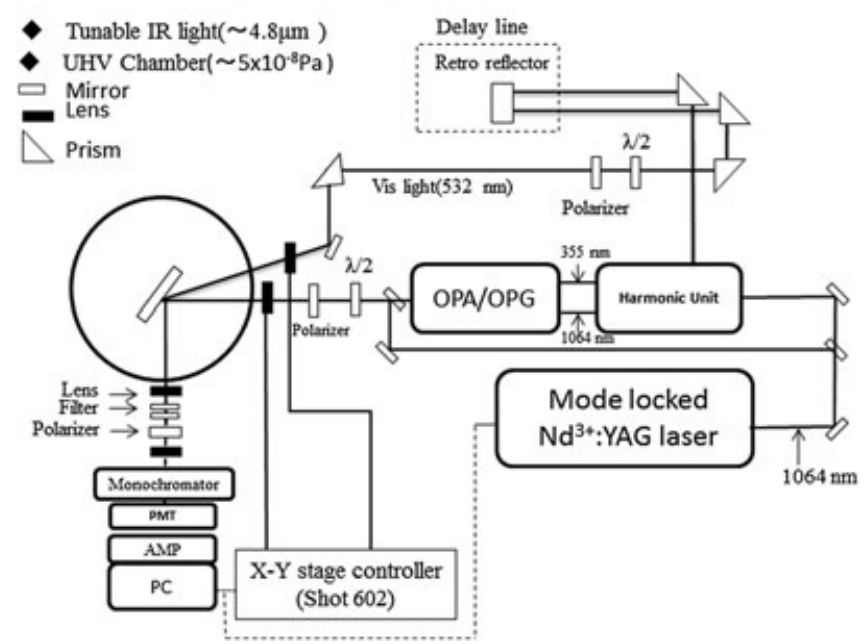

Figure 1. A schematic diagram of the sum frequency generation and second harmonic generation spectroscopic system. as a function of the IR light wavenumber. The SFG spectra were measured from 2060 to $2110 \mathrm{~cm}^{-1}$ with a scanning step of $1 \mathrm{~cm}^{-1}$. The acquisition time for one SFG data point was $\sim 30 \mathrm{~s}$ and that for one SFG spectrum was $\sim 25 \mathrm{~min}$. The typical photon count rate at the SFG peak was $\sim 10$ photons per second, and the energy resolution for SFG spectra was $\sim 2 \mathrm{~cm}^{-z}$. Each measurement was conducted in the polarization combination of $p p p$ (SFG in $p$-polarization, visible in $p$-polarization and $I R$ light in $p$ polarization). After heating for $10 \mathrm{~s}$ at $711 \mathrm{~K}$, the sample was cooled down to room temperature, and the SFG spectrum was taken. This procedure was repeated after total heating time of $20,30,40 \mathrm{~s} .$. and up to $230 \mathrm{~s}$. The coverage of hydrogen was calculated from the resonant SFG signal as a function of the heating time.

In the SHG measurement, we used a fundamental wavelength of $1064 \mathrm{~nm}$ with photon energy $1.17 \mathrm{eV}$ from the same $\mathrm{Nd}^{3+}$ : YAG laser. The incident laser light pulse with an energy of $\sim 380 \mu \mathrm{J} /$ pulse was passed through a half wave plate $(\lambda / 2)$, a Glan polarizer, a color filter, a lens with focal length $f=250 \mathrm{~mm}$, and the $\mathrm{CaF}_{2}$ window of the UHV chamber and finally reached the sample. A color glass $2 \omega$ cut filter was placed between the polarizer and the sample to block unwanted SHG background light from the optics generated prior to the interaction with the sample. The SHG light generated from the sample in the reflective direction was passed through a glass window of the chamber, a focusing lens with focal length $f=300 \mathrm{~mm}$, and $\omega$ cut color glass filters to block the fundamental radiation light beams before coming into the monochromator. $A$ polarizer plate was put before the double monochromator and a photomultiplier to select the polarization of the SHG signal. When the SFG signal became comparable with the background at the lower hydrogen coverage, we switched to SHG measurement and detected the Si dangling bonds. The sample was heated for $50 \mathrm{~s}$ at $711 \mathrm{~K}$ and then cooled down to room temperature, and the SHG signal was taken. This procedure was repeated after total time heating of 230,280,330, and $380 \mathrm{~s} . .$. up to $3880 \mathrm{~s}$. Then we heated the sample for different interval of times and measured SHG signal up to a total heating time of $18330 \mathrm{~s}$.

\section{Results and discussions}

\section{Sum frequency generation response}

In order to confirm the hydrogen desorption order of $\mathrm{H}-\mathrm{Si}$ (111) $1 \times 1$ surfaces with high hydrogen coverage, we investigated the time dependence of isothermal desorption at temperature $711 \mathrm{~K}$ by taking the SFG spectra. Figure 2 shows a typical SFG spec- F2 trum of the $\mathrm{H}-\mathrm{Si}(111) 1 \times 1$ surface at room temperature. The peak

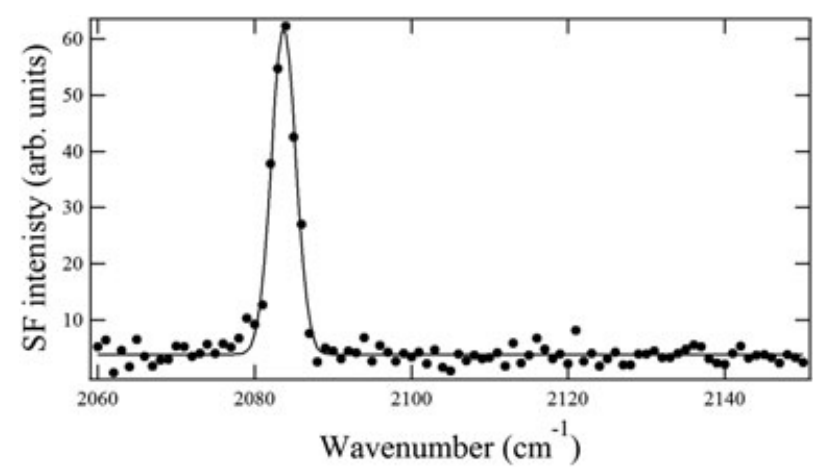

Figure 2. Sum frequency generation spectrum of the $\mathrm{H}-\mathrm{Si}(111) 1 \times 1$ surface at room temperature. A sharp peak appears at $2083.7 \mathrm{~cm}^{-1}$. 
at $2083.7 \mathrm{~cm}^{-1}$ is assigned to the stretching vibration of monohydride on the Si surface. This result is consistent with the literature. ${ }^{[8,11]}$ In general, the hydrogen coverage $\theta$ can be estimated approximately from the peak height of SFG peaks by the following expression as

$$
\theta \propto \sqrt{I_{S F G}} \propto \overleftrightarrow{\chi}(2)
$$

Here, $I_{S F G}$ is the peak height and $\overleftrightarrow{\chi}^{(2)}$ is the nonlinear susceptibility. However, if there is interaction between $\mathrm{Si}-\mathrm{H}$ oscillators, the coverage $\theta$ is not proportional to the value of $\overleftrightarrow{\chi}^{(2)}$ anymore. ${ }^{[12]}$ $\mathrm{Y}$. Miyauchi et al. proved that there was dipole coupling among $\mathrm{Si}-\mathrm{H}$ oscillators on the flat $\mathrm{Si}(111) 1 \times 1$ and calculated the relation between $\theta$ and $\overleftrightarrow{\chi}^{(2)}$ based on the coherent potential approximation method. ${ }^{[13]}$ In our paper, we will calculate the hydrogen coverage with respect to SFG signal $\left(\overleftrightarrow{\chi}^{(2)}\right)$ following Miyauchi's report.

The hydrogen coverage reduction on the Si surface in the desorption kinetics is given by using the Polanyi-Wigner desorption rate equation, ${ }^{[1,8]}$

$$
-\frac{d \theta}{d t}=\vartheta_{d} \theta^{n} e^{-E_{d}} \overline{\overline{\bar{\equiv}}}
$$

Here, $\theta$ is the surface coverage, $\vartheta_{d}$ is the pre-exponential factor, $E_{d}$ is the activation energy for desorption, $R$ is the gas constant $(8.31 \mathrm{~J} / \mathrm{mol} \mathrm{K})$, and $T_{\text {surf }}$ is the surface temperature. When $n=1,1.5$, and 2 , the solutions of desorption rate equation (1) becomes

$$
\begin{gathered}
\text { First-order desorption : } \theta_{t}=\theta_{0} e^{-k_{1} t} \\
\text { 1.5th-order desorption : } \theta_{t}=\theta_{0}\left(1+\sqrt{\theta_{0}} k_{1.5} t\right)^{-2} \\
\text { Second-order desorption : } \theta_{t}=\theta_{0}\left(1+\theta_{0} k_{2} t\right)^{-1}
\end{gathered}
$$

F3 Figure 3 represents the time dependence of the hydrogen coverage at the heating temperature of $711 \mathrm{~K}$. The hydrogen coverages during the isothermal desorption were fitted to the first-order and second-order theoretical curves. The solid dots are hydrogen coverage corresponding to the SFG intensities. The reduction of hydrogen coverage from $1 \mathrm{ML}$ to $0.18 \mathrm{ML}$ in Fig. 3 shows that the second order is the best fitted data with the coverage larger than $0.4 \mathrm{ML}$ as in other reported results. This result is consistent with $\mathrm{M}$. L. Wish et al. ${ }^{[14]}$ using LITD method with the surface temperature of $725 \mathrm{~K}$. In that study, the hydrogen coverage reduced to $0.2 \mathrm{ML}$ after $200 \mathrm{~s}$. In the second-order process, one hydrogen atom leaves a Si atom and diffuses toward another $\mathrm{Si}-\mathrm{H}$ site, and they combine

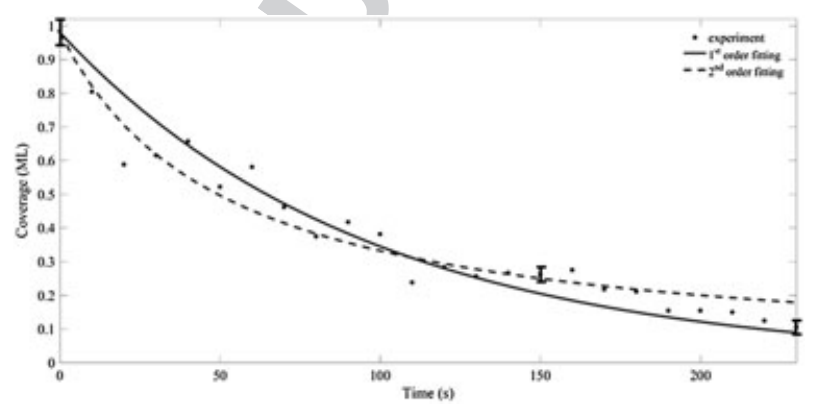

Figure 3. Isothermal hydrogen desorption from the $\mathrm{H}-\mathrm{Si}(111) 1 \times 1$ surface at surface temperatures of $711 \mathrm{~K}$ heated for 10 -s intervals. The solid dots are experimental results, and error bars represent the standard deviations. The solid and dashed lines correspond to the first-order and second-order desorption kinetics. with each other to form a dihydride $\left(\mathrm{Si}-\mathrm{H}_{2}\right)$. For a while, the dihydride state remains. Finally, the hydrogen atoms go beyond the highest potential barrier in the reaction coordinate, associate themselves with each other and desorb from the Si atom. ${ }^{[15]}$ In this way, the hydrogen re-combinative desorption occurs at a single $\mathrm{Si}$ atom. Our investigation confirmed that the hydrogen desorption can be assigned as second order as in the literature ${ }^{[3]}$ in the coverage range of $1.0 \mathrm{ML}-0.18 \mathrm{ML}$.

\section{Second harmonic generation response}

We have used SFG spectra to investigate hydrogen desorption at the high hydrogen coverage from $1 \mathrm{ML}$ to $0.18 \mathrm{ML}$. Because SFG signal is unobservable at lower hydrogen coverage, the SHG spectroscopy is a powerful tool to detect hydrogen coverage below $0.18 \mathrm{ML}$. Reider et al. proved that SHG is sensitive to dangling bonds, especially when hydrogen coverage is lower than $0.3 \mathrm{ML}^{[3]}$

After the SFG experiment, we continued the hydrogen desorption for the same sample as earlier and started the SHG measurement. Figure 4 shows the time dependence of SHG intensity of F4 the $\mathrm{H}-\mathrm{Si}(111) 1 \times 1$ surface when the surface was heated for $50-\mathrm{s}$ intervals at $711 \mathrm{~K}$. In this experiment, we used the polarization configuration $P_{\text {in }} P_{\text {out }}$. The SHG intensity initially increased rapidly as a function of heating time and then gradually saturated when the number of dangling bonds were saturated (Fig. 4).

At the lower coverage, the quenching of the surface susceptibility $\overleftrightarrow{\chi}_{s^{(2)}}(\theta)$ due to adsorbed hydrogen linearly depends on the coverage $(\theta)$ by the following equation.

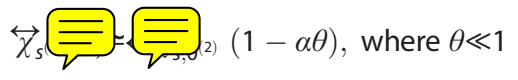

In the case of $\mathrm{H}-\mathrm{Si}(111) 7 \times 7$, the slope is $\alpha \simeq 1.3 .^{[16]}$ Similarly, hydrogen adsorption on $\mathrm{H}-\mathrm{Si}(100) 2 \times 1$ gives a proportionality constant of $\alpha \simeq 3.1 .^{[17]}$ On the other hand, in our case of $\mathrm{H}-\mathrm{Si}$ (111) $1 \times 1$, the proportionality constant of $\alpha \simeq 5.08$ was obtained by using Equation (6) and Fig. 4. Here, $\leftrightarrow \chi_{s^{(2)}}(\theta)$ is proportional to the square root of SHG intensity $\sqrt{I_{S H G}}$, and $\leftrightarrow \chi_{s, 0^{(2)}} \simeq 16.47$ is the susceptibility at $\theta \sim 0 \mathrm{ML}$ obtained from Fig. 4 , and $\alpha$ was determined at $\theta=0.18 \mathrm{ML}$. Figure 5 shows the hydrogen coverage $\mathbf{F 5}$ reduction with respect to the heating time from the $\mathrm{H}-\mathrm{Si}$ (111) $1 \times 1$ surface, calculated via Equation (6). The initial coverage in the SHG measurement was $0.18 \mathrm{ML}$. The hydrogen coverage during the isothermal desorption was fitted with the first-order (1st), intermediate-order (1.5th), and second (2nd)-order theoretical

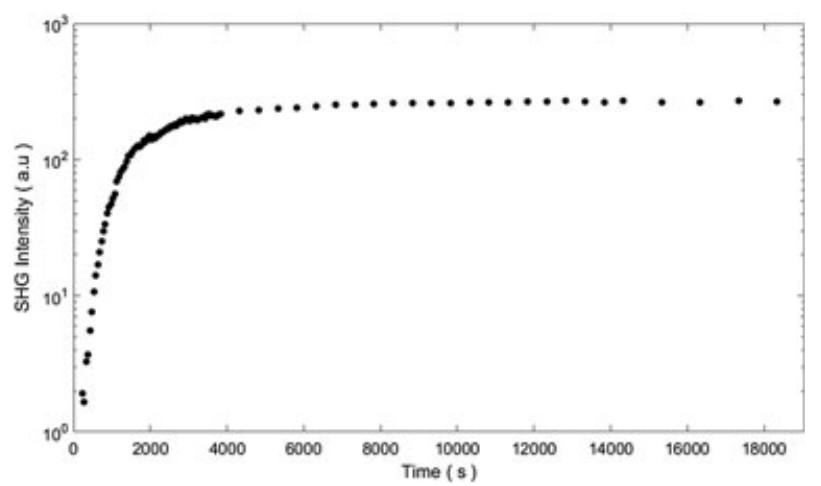

Figure 4. The second harmonic generation (SHG) intensity of the $\mathrm{H} / \mathrm{Si}$ $(111) 1 \times 1$ surface with respect to heating times. Excitation light wavelength is $1064 \mathrm{~nm}$, the polarization of incident light and SHG light was $P_{\text {in }} / P_{\text {out }}$. The solid dots are experimental SHG intensities. 


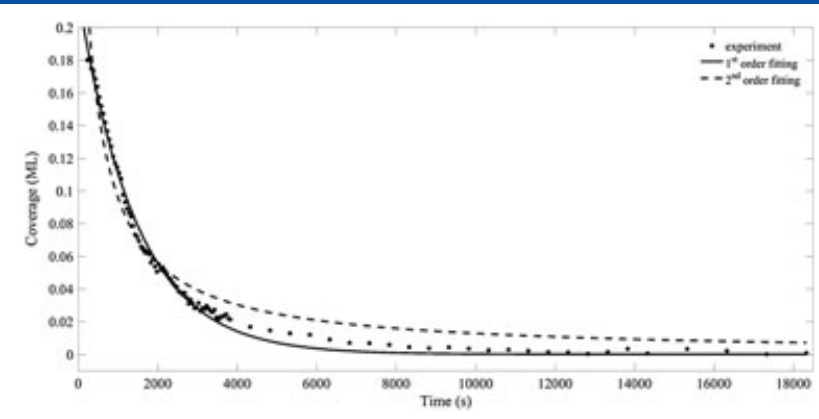

Figure 5. Isothermal hydrogen desorption from the $\mathrm{H}-\mathrm{Si}(111) 1 \times 1$ surface at surface temperatures of $711 \mathrm{~K}$ by using second harmonic generation spectroscopy. The solid dots are experimental hydrogen coverage and solid and dashed lines correspond to the first-order and second-order desorption kinetics.

curves using Equations (3), (4), and (5), respectively. The reduction of hydrogen coverage in Fig. 5 fits the first-order curve best. In the next section, we will try to explain the mechanism of the firstorder desorption.

\section{Hydrogen desorption model}

Isothermal measurements for $\mathrm{H}$ desorption from $\mathrm{Si}$ (111) surfaces have been studied by several authors using different methods. ${ }^{[1,7]}$ They proposed a model that $\mathrm{H}$ desorption from Si (111) was recombinative second-order desorption for monohydride Si (111) surfaces. Indeed, our results confirmed that the hydrogen desorbed in second-order kinetics at the coverage range from $1 \mathrm{ML}$ to $0.18 \mathrm{ML}$. We agree that at the higher coverage of hydrogen than $0.18 \mathrm{ML}$, the hydrogen desorption from the order phase of $1 \times 1$ : $\mathrm{H}$ obeys the second-order desorption. Namely, one hydrogen atom leaves a $\mathrm{Si}$ atom and diffuses toward another $\mathrm{Si}-\mathrm{H}$ site and then they combine to form a dihydride $\left(\mathrm{Si}_{-} \mathrm{H}_{2}\right)$. Finally the molecular hydrogen desorbs from the Si atom.

For the first-order desorption, S. Ciraci et al. proposed that the hydrogen desorption occurs from dihydride species on the Si (100) surface. Two hydrogen atoms re-combined from the two adjacent silicon dihydride species. ${ }^{[18]} \mathrm{M}$. L. Wish et al. proposed another model for the first-order desorption from $\mathrm{Si}(100) 2 \times 1$ surfaces. In this case, hydrogen desorption occurs from two hydrogen atoms paired on the same single dimer of $\mathrm{Si}-\mathrm{Si}^{[14]}$

As seen in Fig. 5, the hydrogen desorbed in the first-order kinetics at the lower coverage from $0.18 \mathrm{ML}-0.0 \mathrm{ML}$ as a function of the heating time. In order to explain the mechanism of the hydrogen desorption, we suggest two candidate models:

Similarly to Y. Morita et al., let us assume that there exist small islands of $\mathrm{Si}$ atoms and hydrogen atoms on the surface. After heating for several hundreds of seconds, these islands becomes crystallized into two-dimensional ((2)D) islands containing one double layer of $\mathrm{Si}(111) 1 \times 1$ atoms terminated by monohydride. ${ }^{[5]}$ During the hydrogen desorption from the surface, each $\mathrm{Si}$ atom from the $2 \mathrm{D}$ island carrying one hydrogen, broke its three back bonds (called $\equiv \mathrm{Si}-\mathrm{H}$ species) and immigrated out of the islands and combined with three Si atoms on the surface in order to reduce the number of dangling bonds. Let us imagine now that there were two types of $\mathrm{Si}-\mathrm{H}$ bonds on the surface. One is remaining monohydride $\mathrm{Si}-\mathrm{H}$ from $1 \times 1$ phase created by hydrogen dosing. The other is $\equiv \mathrm{Si}-\mathrm{H}$ species coming from $2 \mathrm{D}$ islands. When the number of monohydrides $\mathrm{Si}-\mathrm{H}$ on the $1 \times 1$ phase is large ( $1 \mathrm{ML}$ ), the hydrogen desorption on the $1 \times 1$ phase is dominant because the distance between $\mathrm{H}-\mathrm{H}$ atoms on the $1 \times 1$ phase is shorter $3.8 \AA$. Therefore, the second-order desorption is reasonable in spite of the co-existence of small amount of $\equiv \mathrm{Si}-\mathrm{H}$ species. After most of the monohydride $\mathrm{Si}-\mathrm{H}$ desorbed, the coverage becomes lower and the surface contains $\mathrm{H}$ atoms on the $1 \times 1$ : $\mathrm{H}$ phase and from the $\equiv \mathrm{Si}-\mathrm{H}$ species.

As in the paper of Y. Morita et al., ${ }^{[5]}$ we define the coverage of $\equiv \mathrm{Si}$ $\mathrm{H}$ species as $d_{\text {adatom }}(\mathrm{ML})$ and the coverage of remaining monohydride $\mathrm{Si}-\mathrm{H}$ as $d_{\mathrm{SiH}}=\theta(\mathrm{ML})$. The total remaining hydrogen coverage is $d_{\text {adatom }}+d_{\mathrm{SiH}}=\varnothing(\mathrm{ML})$. Then, the desorption order of hydrogen at low coverage becomes smaller than second order following the equation

$$
\frac{d \theta}{d t}=-\frac{k_{d} \theta^{2}}{(1-3 \varnothing)+3 \theta}
$$

From this equation, if $\varnothing=1 / 3$, Equation (7) becomes Equation (3), first-order desorption. This suggestion of Morita et al. is consistent with our experimental results. In our case, when the total coverage of monohydride and $\equiv \mathrm{Si}-\mathrm{H}$ species is $0.18 \mathrm{ML}$, the experimental data is best fitted with the first-order desorption. Therefore, when the surface was heated at $711 \mathrm{~K}$, very small amount of $1 \times 1: \mathrm{H}$ atoms existed within the disordered phase, the number of $\equiv \mathrm{Si}-\mathrm{H}$ species became large, and we expect the high possibility of the $\mathrm{H}-\mathrm{H}$ recombination there.

We suggest another model for the first-order desorption. After hydrogen adsorption, the surface might have the remaining local areas of Si (111)7 $\times 7$ or there might be small surface defects, and thus $\mathrm{Si}-\mathrm{H}_{2}$ and $\mathrm{Si}^{-} \mathrm{H}_{3}$ might exist. G. Schulze et al. suggested that the dihydride and trihydride should desorb from 573 to $770 \mathrm{~K}$ while monohydride should desorb from 680 to $900 \mathrm{~K}^{\left[{ }^{[19]}\right.}$ In our case, the surface was heated at $711 \mathrm{~K}$. In that case, at very low hydrogen coverage, two hydrogen atoms of a $\mathrm{Si}-\mathrm{H}_{2}$ can combine with each other and desorb in the first-order kinetics. Previously, we proved in the SFG spectrum that there was no dihydride and trihydride on the surface. ${ }^{[8]}$ But in that case, the amount of these $\mathrm{SiH}_{2}$ and $\mathrm{SiH}_{3}$ was too small, and SFG could not have detected their vibration. This is just like our result in Fig. 3 that, when the number of monohydride becomes too small due to desorption, SFG could not have detected its vibration. Nevertheless, this suggestion is consistent with another report of A. Ichimiya et al., ${ }^{[20]}$ studied by reflection high energy positron diffraction.

\section{Conclusions}

In this study, we have investigated the hydrogen desorption mechanism from the H-Si (111) $1 \times 1$ by using SFG spectroscopy and SHG spectroscopy. Isothermal desorption was observed at a temperature of $711 \mathrm{~K}$. We suggested that the hydrogen desorption was assigned as second order in the coverage range $1.0 \mathrm{ML}-0.18 \mathrm{ML}$ by SFG, and the hydrogen desorption was assigned as first order in the coverage range $0.18 \mathrm{ML}-0.0 \mathrm{ML}$ by SHG. We have not yet calculated the hydrogen desorption activation energy for the first-order desorption from $0.18 \mathrm{ML}$ to $0.0 \mathrm{ML}$ in this experiment. So further experiments at different temperatures are required. From that result, the mechanism of the first-order desorption will be determined.

\section{Acknowledgements}

We would like to thank Prof. Emeritus A. Ichimiya of Nagoya University Japan for valuable advice. 


\section{References}

[1] B. G. Koehler, C. H. Mak, D. A. Arthur, J Chem Phys, 1988, 89(3), 1709.

[2] K. Sinniah, M. G. Sherman, L. B. Lewis, Phys Rev Lett, 1989, 62, 567.

[3] G. A. Reider, U. Hofer, T. F. Heinz, J Chem Phys, 1991, 94, 4080-4083.

[4] M. Niwano, M. Terashi, J. Kuge, Surf Sci, 1999, 420, 6.

[5] Y. Morita, K. Miki, H. Tokumoto, Sur Sci, 1995, 325, 21.

[6] M. C. Flowers, N. B. H. Jonathan, Y. Liu, J Chem Phys, 1995, 102, 1034

[7] P. Gupta, V. L. Colvin, S. M. George, Phys Rev B, 1988, 37(14), 8234.

[8] K. T. T. Hien, Y. Miyauchi, G. Mizutani, Surf Interface Anal, 2012, 44, 662-665.

[9] $R_{2}$ Culbertson, L. Feldman, P. Silverman, J Vac Sci Technol, 1982, 20, 868.

[10] B. G. Koehler, S. M. George, Surf Sci, 1991, 248(1-2), 158-172.

[11] M. Y. Mao, P. B. Miranda, D. S. Kim, Phys Rev B, 2001, 64, 035415.
[12] A. Bandara, S. Katano, J. Kubota, et at, Chem Phys Lett, 1998, 290(1-3), 261-267.

[13] Y. Miyauchi, H. Chuat, G. Mizutani, Surf Sci, 2013, 614, 24-29.

[14] M. L. Wish, B. G. Koehler, P. Gupta, Surf Sci, 1991, 258, 166-176.

[15] A. Vittadini, A. Sellłoni, Surf Sci, 1997, 383, L779-L784.

[16] U. Hofer, Appl Phys A, 1996, 63, 533-547.

[17] P. Bratu, K. L. Kompa, U. H. Ofer, Chem Phys Lett, 1996, 251(1).

[18] S. Ciraci, I. Batra, Surf Sci, 1986, 178, 80 .

[19] G. Schulze, M. Henzler, Surf Sci, 1983, 124, 336-350.

[20] A. Kawasuso, M. Yoshikawa, K. Kojima, S. Okada, A. Ichimiya, Physical Review B, 2000, 61(3), 2102-2106. 


\section{Author Query Form}

\section{Journal: Surface and Interface Analysis}

\section{Article: sia_6099}

Dear Author,

During the copyediting of your paper, the following queries arose. Please respond to these by annotating your proofs with the necessary changes/additions.

- If you intend to annotate your proof electronically, please refer to the E-annotation guidelines.

- If you intend to annotate your proof by means of hard-copy mark-up, please use the standard proofing marks. If manually writing corrections on your proof and returning it by fax, do not write too close to the edge of the paper. Please remember that illegible mark-ups may delay publication.

Whether you opt for hard-copy or electronic annotation of your proofs, we recommend that you provide additional clarification of answers to queries by entering your answers on the query sheet, in addition to the text mark-up.

\begin{tabular}{|c|l|l|}
\hline Query No. & \multicolumn{1}{|c|}{ Query } & \multicolumn{1}{|c|}{ Remark } \\
\hline Q1 & Production Editor: Please provide manuscriptRevised date. & 7 Dec., 2015 \\
\hline Q2 & AUTHOR: Please check that affiliations are correct. & OK \\
\hline Q3 & $\begin{array}{l}\text { AUTHOR: ultra-high vacuum. Is this the correct definition for UHV? Please change if this } \\
\text { is incorrect. }\end{array}$ & $\begin{array}{l}\text { OK, but the article } \\
\text { Was changed }\end{array}$ \\
\hline Q4 & $\begin{array}{l}\text { AUTHOR: optical parametric generator. Is this the correct definition for OPG? Please } \\
\text { change if this is incorrect. }\end{array}$ & OK \\
\hline Q5 & $\begin{array}{l}\text { AUTHOR: optical parametric amplifier. Is this the correct definition for OPA? Please } \\
\text { change if this is incorrect. }\end{array}$ & OK \\
\hline Q6 & AUTHOR: Please provide the list of all authors; no et al for Reference 12. & OK \\
\hline Q7 & AUTHOR: Please provide the page number for Reference 17. & OK \\
\hline Q8 & $\begin{array}{l}\text { AUTHOR: Please confirm that given names (red) and surnames/family names (green) } \\
\text { have been identified correctly. }\end{array}$ & OK \\
\hline
\end{tabular}


Required software to e-Annotate PDFs: Adobe Acrobat Professional or Adobe Reader (version 7.0 or above). (Note that this document uses screenshots from Adobe Reader $\mathrm{X}$ )

The latest version of Acrobat Reader can be downloaded for free at: http://get.adobe.com/uk/reader/

Once you have Acrobat Reader open on your computer, click on the Comment tab at the right of the toolbar:

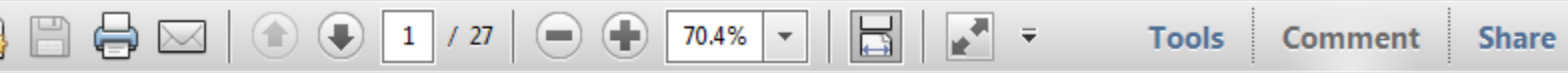

This will open up a panel down the right side of the document. The majority of tools you will use for annotating your proof will be in the Annotations section, pictured opposite. We've picked out some of these tools below:

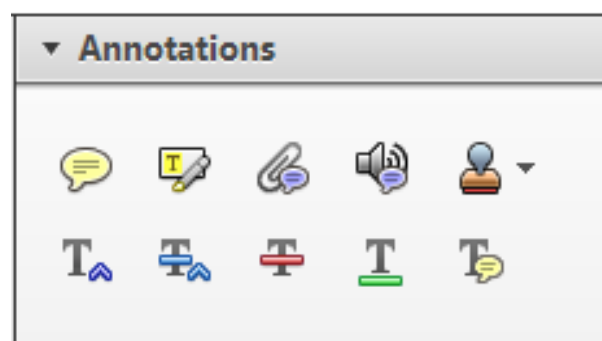

1. Replace (Ins) Tool - for replacing text.

Strikes a line through text and opens up a text box where replacement text can be entered.

How to use it

- Highlight a word or sentence.

- Click on the Replace (Ins) icon in the Annotations section.

- Type the replacement text into the blue box that appears.

Idard tramework for the analysis of $\mathrm{m}$ icy-Nevertheless, it also led to exog،

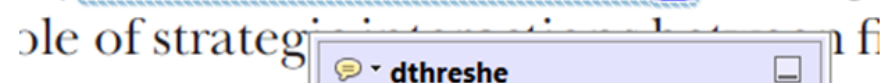
aber of comp 08/06/2011 15:58:17 is that the s1 nain compo: be level, are exc nc

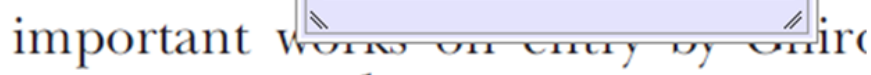
M heneferth) ${ }^{1}$ we anen the "hlarl $\mathrm{l}$

3. Add note to text Tool - for highlighting a section to be changed to bold or italic.

T Highlights text in yellow and opens up a text box where comments can be entered.

\section{How to use it}

- Highlight the relevant section of text.

- Click on the Add note to text icon in the Annotations section.

- Type instruction on what should be changed regarding the text into the yellow box that appears.

namic responses of mark ups ent with the VAR evidence

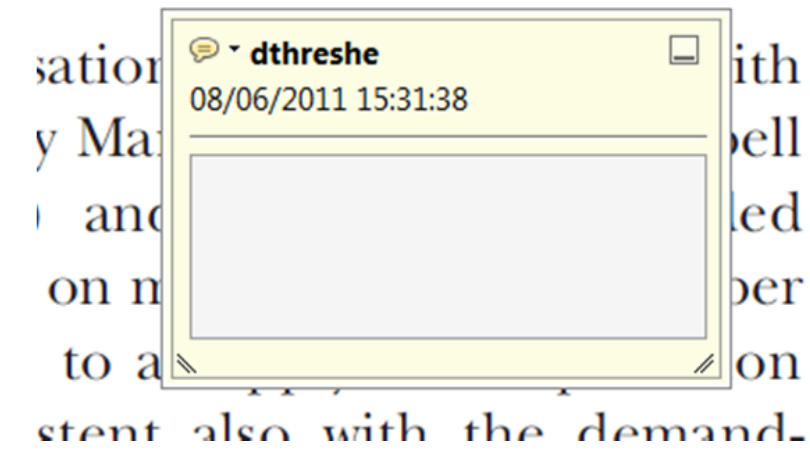

2. Strikethrough (Del) Tool - for deleting text.

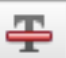

Strikes a red line through text that is to be deleted.

How to use it

- Highlight a word or sentence.

- Click on the Strikethrough (Del) icon in the Annotations section.

there is no room tor extra prohts al c ups are zero and the number of ret) values are not determined by Blanchard and Kiyotaki (1987), sfect competition in general equilil ts of aggregate demand and supply lassical framework assuming monol eph on evorenous number of firme

4. Add sticky note Tool - for making notes at specific points in the text.

Marks a point in the proof where a comment needs to be highlighted.

How to use it

- Click on the Add sticky note icon in the Annotations section.

- Click at the point in the proof where the comment should be inserted.

- Type the comment into the yellow box that appears.

iaisu airu suppiy sirucks. hivsl ui

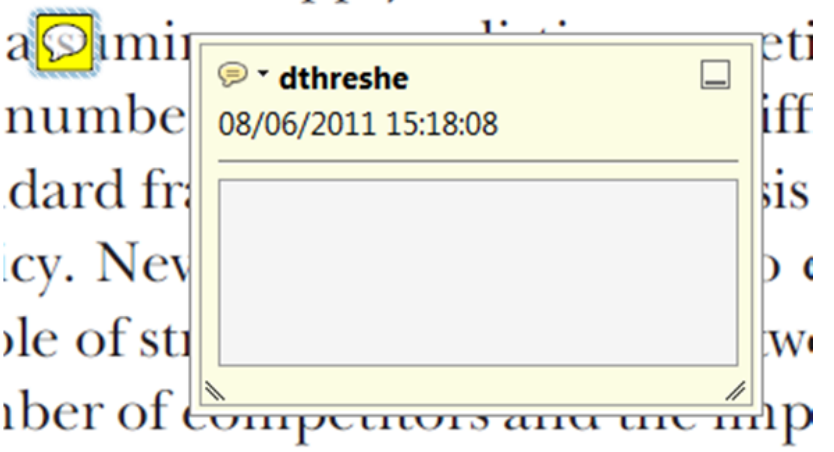

is that the structure of the secto. 
5. Attach File Tool - for inserting large amounts of text or replacement figures.

Inserts an icon linking to the attached file in the appropriate pace in the text.

How to use it

- Click on the Attach File icon in the Annotations section.

- Click on the proof to where you'd like the attached file to be linked.

- Select the file to be attached from your computer or network.

- Select the colour and type of icon that will appear in the proof. Click OK.

E N D

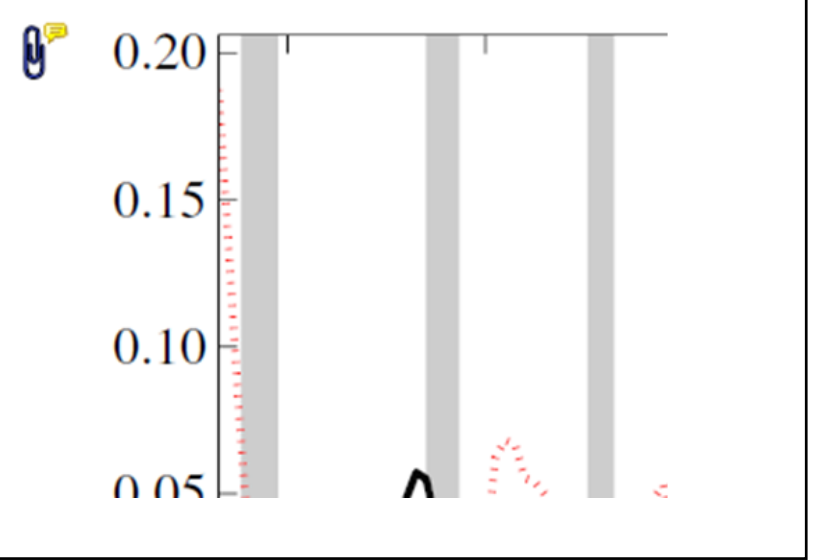

6. Add stamp Tool - for approving a proof if no corrections are required.

- Inserts a selected stamp onto an appropriate place in the proof.

\section{How to use it}

- Click on the Add stamp icon in the Annotations section.

- $\quad$ Select the stamp you want to use. (The Approved stamp is usually available directly in the menu that appears).

- Click on the proof where you'd like the stamp to appear. (Where a proof is to be approved as it is, this would normally be on the first page).

or the business cycie, starting with the on perfect competition, constant ret

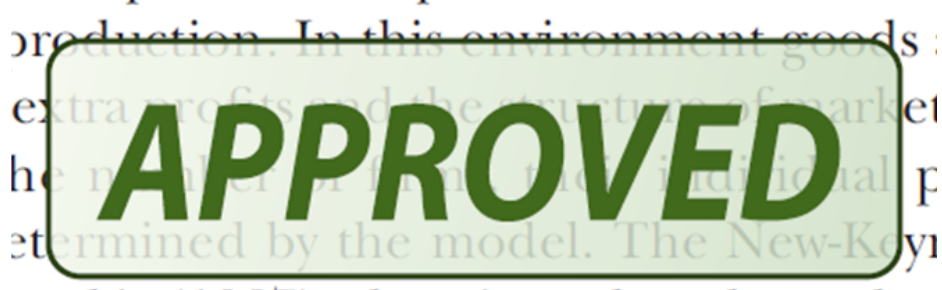
otaki (1987), has introduced produc general equilibrium models with nomin:

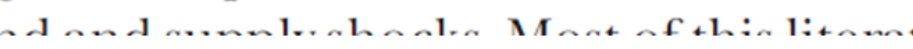

- Drawing Markups

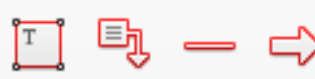

$0 \bigcirc \sqrt{6} \otimes$

\section{How to use it}

- Click on one of the shapes in the Drawing Markups section.

- Click on the proof at the relevant point and draw the selected shape with the cursor.

- To add a comment to the drawn shape, move the cursor over the shape until an arrowhead appears.

- Double click on the shape and type any text in the red box that appears.
7. Drawing Markups Tools - for drawing shapes, lines and freeform annotations on proofs and commenting on these marks.

Allows shapes, lines and freeform annotations to be drawn on proofs and for comment to be made on these marks.

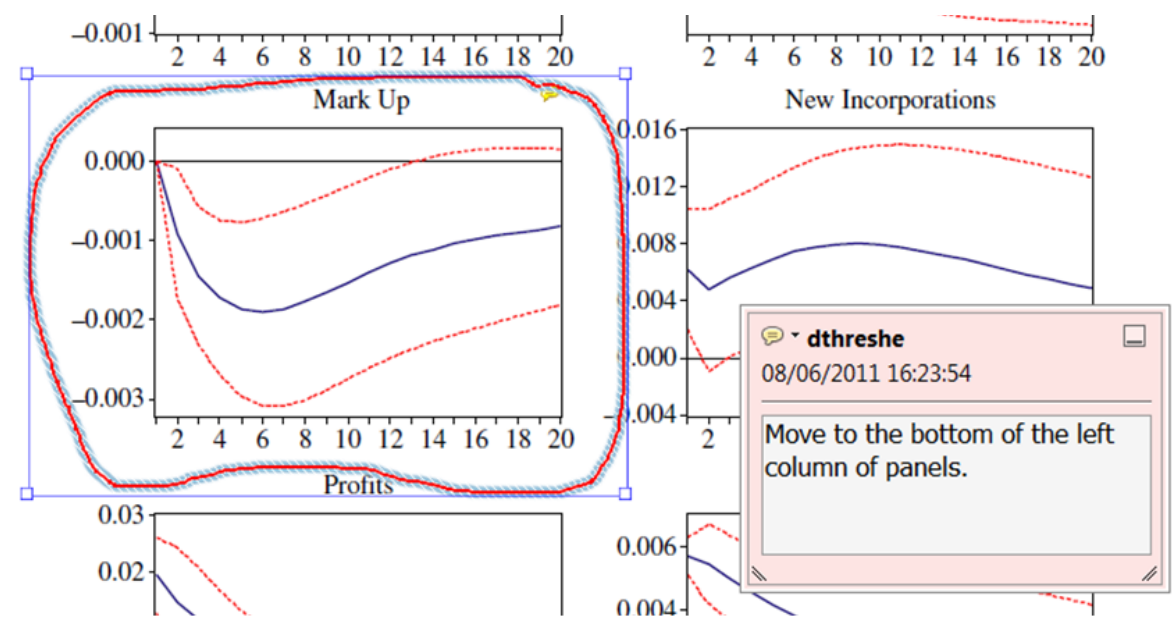

For further information on how to annotate proofs, click on the Help menu to reveal a list of further options:

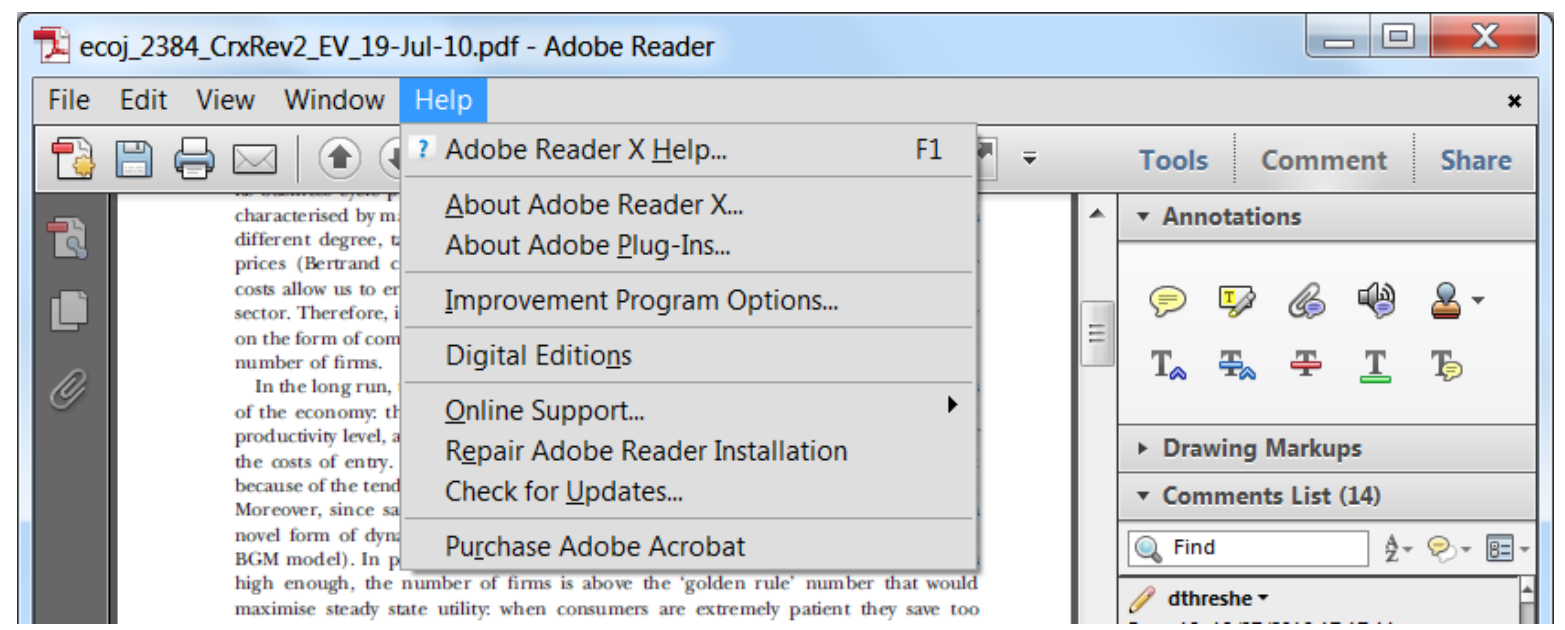

